\title{
Front propagation in anomalous diffusive media governed by time-fractional diffusion
}

\author{
Andrea Mentrelli ${ }^{\mathrm{a}, \mathrm{b}}$, Gianni Pagnini ${ }^{\mathrm{b}, \mathrm{c}}$ \\ ${ }^{a}$ Department of Mathematics $\&$ \\ Alma Mater Research Center on Applied Mathematics - $(A M)^{2}$, \\ University of Bologna, via Saragozza 8, 40123 Bologna, Italy \\ ${ }^{b}$ BCAM - Basque Center for Applied Mathematics, \\ Alameda de Mazarredo 14, 48009 Bilbao, Basque Country - Spain \\ ${ }^{c}$ Ikerbasque - Basque Foundation for Science, \\ Calle de María Díaz de Haro 3, 48013 Bilbao, Basque Country - Spain
}

\begin{abstract}
In this paper, a multi-dimensional model is proposed to study the propagation of random fronts in media in which anomalous diffusion takes place. The front position is obtained as the weighted mean of fronts calculated by means of the level set method, using as weight-function the probability density function which characterizes the anomalous diffusion process. Since anomalous diffusion is assumed to be governed by a time-fractional diffusion equation, its fundamental solution is the required probability density function. It is shown that this fundamental solution can be expressed in the multi-dimensional case in terms of the well-known $\mathcal{M}$-Wright/Mainardi function, as in the one-dimensional case. Making use of this representation for the practical purpose of numerical evaluation, the propagation of random fronts in two-dimensional subdiffusive media is discussed and investigated.
\end{abstract}

Keywords: random front propagation, Level Set Method, multidimensional time-fractional diffusion, $\mathcal{M}$-Wright/Mainardi function

2010 MSC: 60G22, 60K37, 35F21, 65M08

Email addresses: andrea.mentrelli@unibo.it (Andrea Mentrelli), gpagnini@bcamath.org (Gianni Pagnini)

Preprint submitted to Journal of Computational Physics

December 2, 2014 


\section{Introduction}

Front propagation is of interest in several fields of applied science. In many cases, the propagating front is embedded in a medium characterized by a random motion and then by a diffusion process. In typical diffusion processes the mean

5 square displacement of particles is a linear function of time. Nevertheless in some complex media diffusion processes show a non-linear relationship to time, as for example in biological systems [1,2,3], in fluids and plasmas [4] and in other systems [5]. In the latter case, the diffusion process is known as anomalous diffusion $[6,2,1,7]$.

The aim of the present paper is to extend to the multi-dimensional case a recently developed one-dimensional model useful for the study of the propagation of fronts in media in which anomalous diffusion takes place [8].

The main items of the proposed approach are:

- the average front position is modelled by means of the level set method (LSM) [9];

- the spreading of interface particles around the average front is described by the probability density function (PDF) that characterizes the diffusive process;

- the effective front is obtained as the weighted mean of average fronts, by using as weight-function the PDF of particle spreading.

This approach to the study of random fronts was first proposed - in a context in which anomalous diffusion was not involved - to investigate the evolution of the burnt mass fraction in turbulent premixed combustion [10], and has recently been applied to wildland fire propagation $[11,12,13,14,15]$. In these studies, the chosen PDF was the Gaussian density function $[10,11,12,13,14,15]$, or a convolution of the Gaussian with an other PDF $[13,14,15]$. In the present study, since anomalous diffusion is assumed to be modelled by the time-fractional diffusion equation, i.e. a diffusion-like equation in which the first order derivative 
in time is replaced by a derivative operator of fractional order, the required PDF to be used as weight-function is the fundamental solution, or Green's function, of this family of fractional partial differential equations.

It is well known that in the one-dimensional case that Green's function is related to the $\mathcal{M}$-Wright/Mainardi function $[16,17,18]$, which tends to the Gaussian function as the fractional order of the time derivative tends to one.

35 The $\mathcal{M}$-Wright/Mainardi function is a well known function for the evaluation of which robust numerical algorithms exist [19]. However, the fundamental solution of the multidimensional time-fractional diffusion equation has a cumbersome representation [20] so that it is rather complicated to deal with for any practical purpose. Due to this difficulty, the extension discussed in this paper

40 of the one-dimensional case investigated in [8] to the multi-dimensional cases is one of the highlights of the paper.

In the following it is shown that, by using an integral representation formula of the $\mathcal{M}$-Wright/Mainardi function [21], which is based on the one-dimensional Gaussian PDF, Green's function for multi-dimensional cases (including the most interesting two- and three-dimensional cases) can be represented by the same integral formula involving the corresponding multi-dimensional Gaussian PDF. This formula strongly simplifies the representation of Green's function provided in $[20]$.

As previously mentioned, this PDF is integrated in the proposed approach that is based on the well-established LSM.

The LSM $[22,9]$ is one of the most widely used and successful tools for computing and analyzing the motion of a front and it has been adopted in many different problems, including turbulent premixed combustion [23], wildland fire propagation [24], groundwater infiltration [25], biology [26] and material science $55 \quad[27]$.

In the framework of the LSM, the location of the propagating front is obtained as a level set (for example the zero level set) of an auxiliary function solution of a Hamilton-Jacobi equation known as level set equation. 
In this setting, sharp gradients and cusps can be easily accounted for and larly useful to handle problems in which the speed of the evolving interface is dependent on the interface properties, such as curvature and normal direction, as well as on the boundary conditions at the interface location. Hence, it is suitable for problems in which the topology of the evolving interface changes in time, like when topological merging and splitting occur.

In addition to these advantages, one key-feature of the level set method framework is that it allows to easily improve the physical model by progressively including more detailed interface effects that might influence the front propagation, as the dispersion effects of interface particles due to diffusive phe70 nomena considered in this study.

The formalism of the proposed approach can be related to the Smoothed Particle Hydrodynamics (SPH) [28]. This framework is characterized by the robustness of its numerical implementation and it is well suited for many practical problems as fluid-structure interactions. However, the typical problems 75 of SPH, namely the choice of the kernel function and of the smoothing length, are here avoided because they are straightforwardly determined by the particle PDF. This can be considered a positive feature of the present approach.

The presented approach can be related also to approaches used in turbulent premixed combustion, namely the LSM/G-equation [23] or the so-called turbulent flame closure model first proposed by Zimont [29, 30]. A discussion about how these frameworks are related to the one presented here might be found in [10].

Furthermore, the present approach is also connected to the so-called Stochastic Level Set Method (SLSM), developed and adopted in computer vision. Typical problems in this research area consist in recovering a certain surface or region through a shape optimization framework. However, classical methods suffer from being sometimes stuck in local minima. To overcome this difficulty, the SLSM has been developed. This method combines stochastic motion and 
the classical LSM $[31,32,33]$ and adopts a decision mechanism. The SLSM is quite close to the approach here proposed, as in both models the interface location is obtained by solving the level set equation with a suitably randomized front speed. However, the final aims of the two methods are different, and so is the mathematical construction. In fact, in typical computer vision problems the final aim is not to compute the average shape after a large number of independent realizations, but to recover a certain shape with the frontline upon the derivation of a proper stochastic differential equation. In the present study, the interest is indeed focused on the average properties of the processes for applications in physics and engineering. Even if it could be possible to have average properties of the process obtained with the SLSM by performing the ensemble average of many independent realizations, the approach here proposed uses physical arguments to derive the PDF of the interface particle displacement instead of focusing on the selection of the correct stochastic differential equation, so it can be regarded as the averaged counterpart of the method adopted in computer vision. Despite this difference in the two models, the large available literature regarding SLSM is an important guideline for future derivation of stochastic models within the approach proposed here.

In analogy with studies on diffusion processes that describe the properties of the underlying stochastic processes by the particle PDF and averaged quantities, here the motion of random fronts is described is well in terms of an ensemble average resulting in the mentioned effective front.

The paper is organized as follows. Section 2 contains the description of the LSM-based model used to study the propagation of a front embedded in a diffusive environment. In Section 3, it is recalled the time-fractional diffusion equation governing the dispersion of the interface particles and its solutions in the one-, two- and three-dimensional cases are discussed. In particular, it is shown a new representation of the Green's functions of the time-fractional diffusion equation, based on the Gaussian PDF, which can conveniently be used for computational purposes. Section 4 contains a discussion of the numerical 
methodology adopted for the calculation of Green's functions as well as the techniques adopted for the computation of the effective average front. Numerical results are also presented, including plots of the $\mathcal{M}$-Wright/Mainardi function for the one-, two- and three-dimensional cases as well as a selection of numerical results concerning two-dimensional front propagation in media in which anomalous diffusion takes place, restricting the attention to the case of subdiffusive processes, i.e. those processes whose particle displacement variance grows less than linearly in time. Finally, in Section 5, conclusions are drawn and some future developments are proposed.

\section{Modelling approach}

Let us consider a propagating interface composed of a sufficiently large numinitial instant $t=0$, and let $\mathbf{X}^{\omega}\left(t, \overline{\mathbf{x}}_{0}\right)$ be the $\omega$-realization of the trajectory of a particle $P_{0}$ located in $\overline{\mathbf{x}}_{0}$ at $t=0$, such that $\mathbf{X}^{\omega}\left(0, \overline{\mathbf{x}}_{0}\right)=\overline{\mathbf{x}}_{0}$. By using statistical mechanics formalism [34], the trajectory of particle $P_{0}$ is described by the one-particle density function $p_{d}^{\omega}(\mathbf{x} ; t)=\delta\left(\mathbf{x}-\mathbf{X}^{\omega}\left(t, \overline{\mathbf{x}}_{0}\right)\right)$, where $\delta(\mathbf{x})$ is

Let $N_{\omega}$ be the number of the independent realizations indexed by $\omega$, denoting by $\langle\cdot\rangle$ the ensemble average, then the PDF of the displacement of the interface particle $P_{0}$ is written as

$$
p_{d}\left(\mathbf{x} ; t \mid \overline{\mathbf{x}}_{0}\right)=\frac{1}{N_{\omega}} \sum_{\omega=1}^{N_{\omega}} \delta\left(\mathbf{x}-\mathbf{X}^{\omega}\left(t, \overline{\mathbf{x}}_{0}\right)\right)=\left\langle\delta\left(\mathbf{x}-\mathbf{X}^{\omega}\left(t, \overline{\mathbf{x}}_{0}\right)\right)\right\rangle,
$$

that can be understood as the PDF counterpart of the empirical distribution function [35]. If the density of particles embodying the interface is assumed to be constant during the interface evolution, then an incompressibility-like condition can be stated from the Jacobian $J$ of the transformation $\overline{\mathbf{x}} \equiv \overline{\mathbf{x}}\left(t, \overline{\mathbf{x}}_{0}\right)$ : $J=d \overline{\mathbf{x}}_{0} / d \overline{\mathbf{x}}=1$. Finally, in terms of the average position $\overline{\mathbf{x}}$ of particle $P_{0}$, it holds

$$
p_{d}(\mathbf{x} ; t \mid \overline{\mathbf{x}})=\left\langle\delta\left(\mathbf{x}-\mathbf{X}^{\omega}(t, \overline{\mathbf{x}})\right)\right\rangle
$$


Let $\Gamma$ be a simple closed surface, or an ensemble of simple non-intersecting closed surfaces, that represents the propagating interface under consideration and let $\Omega$ be the region bounded by $\Gamma$. In the case of an interface $\Gamma$ made of more than one closed surface, the domain $\Omega$ is not simply connected, resulting in more than one bounded regions independently evolving. Moreover, let $\gamma$ : $\mathcal{S} \times\left[0,+\infty\left[\rightarrow \mathbb{R}\right.\right.$ be a function defined on the domain of interest $\mathcal{S} \subseteq \mathbb{R}^{d}$ such that the level set $\gamma(\mathbf{x}, t)=\gamma_{*}$ coincides with the evolving front, i.e. $\Gamma(t)=$ $\left\{\mathbf{x} \in \mathcal{S} \mid \gamma(\mathbf{x}, t)=\gamma_{*}\right\}$. In the case of $\Gamma$ being an ensemble of $n$ surfaces, the ensemble of the $n$ interfaces is considered as interface.

In a deterministic setting, the level set function $\gamma(\mathbf{x}, t)$ may be written as

$$
\gamma(\mathbf{x}, t)=\int_{\mathcal{S}} \gamma(\overline{\mathbf{x}}, t) \delta(\mathbf{x}-\overline{\mathbf{x}}) d \overline{\mathbf{x}}
$$

and the subsets of the domain $\mathcal{S}$ corresponding to the interface $\Gamma$ and to the region $\Omega$ enclosed by $\Gamma$ (which represent, respectively, the interface and the domain bounded by it) may be conveniently described by two indicator functions $\mathcal{I}_{\Gamma}, \mathcal{I}_{\Omega}: \mathcal{S} \times[0,+\infty[\rightarrow\{0,1\}$ defined as follows:

$$
\mathcal{I}_{\Gamma}(\mathbf{x}, t)= \begin{cases}1, & \text { if } \gamma(\mathbf{x}, t)=\gamma_{*} \\ 0, & \text { elsewhere }\end{cases}
$$

and

$$
\mathcal{I}_{\Omega}(\mathbf{x}, t)= \begin{cases}1, & \text { if } \gamma(\mathbf{x}, t) \leqslant \gamma_{*} \\ 0, & \text { elsewhere. }\end{cases}
$$

The indicator functions at time $t=0$, i.e. $\mathcal{I}_{\Gamma}(\mathbf{x}, t=0)$ and $\mathcal{I}_{\Omega}(\mathbf{x}, t=0)$, are denoted in the following as $\mathcal{I}_{\Gamma_{0}}(\mathbf{x})$ and $\mathcal{I}_{\Omega_{0}}(\mathbf{x})$, respectively.

In a random setting, the level set function corresponding to the $\omega$-realization $\gamma^{\omega}(\mathbf{x}, t)$, which embeds the random front $\Gamma^{\omega}$, for each realization is written as

$$
\gamma^{\omega}(\mathbf{x}, t)=\int_{\mathcal{S}} \gamma(\overline{\mathbf{x}}, t) \delta\left(\mathbf{x}-\mathbf{X}^{\omega}(t, \overline{\mathbf{x}})\right) d \overline{\mathbf{x}}
$$


and, accordingly, $\mathcal{I}_{\Gamma}$ and $\mathcal{I}_{\Omega}$ are replaced by random indicator functions $\mathcal{I}_{\Gamma^{\omega}}, \mathcal{I}_{\Omega^{\omega}}$ : $\mathcal{S} \times[0,+\infty[\rightarrow\{0,1\}$ defined as follows:

$$
\begin{aligned}
\mathcal{I}_{\Gamma^{\omega}}(\mathbf{x}, t) & =\int_{\mathcal{S}} \mathcal{I}_{\Gamma_{0}}\left(\overline{\mathbf{x}}_{0}\right) \delta\left(\mathbf{x}-\mathbf{X}^{\omega}\left(t, \overline{\mathbf{x}}_{0}\right)\right) d \overline{\mathbf{x}}_{0} \\
& =\int_{\Gamma_{0}} \delta\left(\mathbf{x}-\mathbf{X}^{\omega}\left(t, \overline{\mathbf{x}}_{0}\right)\right) d \overline{\mathbf{x}}_{0} \\
& =\int_{\Gamma(t)} \delta\left(\mathbf{x}-\mathbf{X}^{\omega}(t, \overline{\mathbf{x}})\right) d \overline{\mathbf{x}}
\end{aligned}
$$

and

$$
\begin{aligned}
\mathcal{I}_{\Omega^{\omega}}(\mathbf{x}, t) & =\int_{\mathcal{S}} \mathcal{I}_{\Omega_{0}}\left(\overline{\mathbf{x}}_{0}\right) \delta\left(\mathbf{x}-\mathbf{X}^{\omega}\left(t, \overline{\mathbf{x}}_{0}\right)\right) d \overline{\mathbf{x}}_{0} \\
& =\int_{\Omega_{0}} \delta\left(\mathbf{x}-\mathbf{X}^{\omega}\left(t, \overline{\mathbf{x}}_{0}\right)\right) d \overline{\mathbf{x}}_{0} \\
& =\int_{\Omega(t)} \delta\left(\mathbf{x}-\mathbf{X}^{\omega}(t, \overline{\mathbf{x}})\right) d \overline{\mathbf{x}} .
\end{aligned}
$$

In the case of a non-deterministic interface, an effective indicator of the region surrounded by a random front, $\varphi_{e}(\mathbf{x}, t): \mathcal{S} \times[0,+\infty[\rightarrow[0,1]$, was defined in [10] (in the context of modelling of the burnt mass fraction in turbulent premixed combustion) as follows:

$$
\begin{aligned}
\varphi_{e}(\mathbf{x}, t)=\left\langle\mathcal{I}_{\Omega^{\omega}}(\mathbf{x}, t)\right\rangle & =\left\langle\int_{\Omega(t)} \delta\left(\mathbf{x}-\mathbf{X}^{\omega}(t, \overline{\mathbf{x}})\right) d \overline{\mathbf{x}}\right\rangle \\
& =\int_{\Omega(t)}\left\langle\delta\left(\mathbf{x}-\mathbf{X}^{\omega}(t, \overline{\mathbf{x}})\right)\right\rangle d \overline{\mathbf{x}} \\
& =\int_{\Omega(t)} p_{d}(\mathbf{x} ; t \mid \overline{\mathbf{x}}) d \overline{\mathbf{x}} .
\end{aligned}
$$

It should be noted that the effective indicator $\varphi_{e}(\mathbf{x}, t)$ is not an indicator function in the classical sense. Actually, by using terminology from fuzzy logic, it is a membership function, its range being the compact interval $[0,1]$ rather than the discrete set $\{0,1\}$. Despite this, the concept of probability which led to Eq. (2) should not be confused with the concept of degree of truth (typical of fuzzy logic), then $\varphi_{e}(\mathbf{x}, t)$ is classified as an indicator function. Since the range of the effective indicator $\varphi_{e}(\mathbf{x}, t)$ is the compact interval $[0,1]$, a criterion to mark the effective surrounded region $\Omega_{e}$ has to be stated. The region $\Omega_{e}$ is 

value arbitrarily fixed $\varphi_{e}^{t h}$, i.e. $\Omega_{e}(t)=\left\{\mathbf{x} \in \mathcal{S} \mid \varphi_{e}(\mathbf{x}, t) \geqslant \varphi_{e}^{t h}\right\}$.

Making use of the indicator function $\mathcal{I}_{\Omega}$ defined in Eq. (1), Eq. (2) can be rewritten as:

$$
\varphi_{e}(\mathbf{x}, t)=\int_{\mathcal{S}} \mathcal{I}_{\Omega}(\overline{\mathbf{x}}, t) p_{d}(\mathbf{x} ; t \mid \overline{\mathbf{x}}) d \overline{\mathbf{x}}
$$

In the deterministic case, i.e. $\mathbf{X}^{\omega}(t, \overline{\mathbf{x}})=\overline{\mathbf{x}}(t)$ for all realizations, it turns out that $p_{d}(\mathbf{x} ; t \mid \overline{\mathbf{x}})=\delta(\mathbf{x}-\overline{\mathbf{x}}(t))$, and from Eq. (3) it is obtained $\varphi_{e}(\mathbf{x}, t)=\mathcal{I}_{\Omega}(\mathbf{x}, t)$. Eq. (3) is similar to the basic equation of SPH method [28], however, as stated in the Introduction, the choices of the kernel function and of the smoothing length are avoided because they are straightforwardly determined by the particle PDF $p_{d}(\mathbf{x} ; t \mid \overline{\mathbf{x}})$.

In conclusion, the indicator function $\mathcal{I}_{\Omega}(\mathbf{x}, t)$ marks the region $\Omega(t)$ that is bounded by an interface propagating according to the average trajectory $\overline{\mathbf{x}}(t)$. Recalling the definition of $\mathcal{I}_{\Omega}(\mathbf{x}, t)$ in Eq. (1), the evolution of the level set function $\gamma(\mathbf{x}, t)$ is governed by the following Hamilton-Jacobi equation

$$
\frac{\partial \gamma}{\partial t}=\mathcal{V}(\mathbf{x}, t)\|\nabla \gamma\|
$$

where $\gamma_{0}$ is the initial field embedding the interface $\Gamma$ at $t=0, \Gamma_{0} \equiv \Gamma(t=0)$.

It is worth mentioning here that an alternative representation of the dynamics of the propagating front is possible whenever velocity $\mathcal{V}(\mathbf{x}, t)$ is constant in time and strictly positive (or strictly negative). In this case, let $\tau(\mathbf{x})$ be the arrival time function that represents the temporal instant at which the front reaches the point $\mathbf{x}$, then $\|\nabla \tau\|$ is the rate of change of the arrival time with respect to the change in the front distance, i.e.

$$
\|\nabla \tau\|=\frac{1}{\mathcal{V}}, \quad \tau(\mathbf{x})=0 \quad \forall \mathbf{x} \in \Gamma_{0} .
$$

Equation (5) is known as eikonal equation and it is a time-independent version of the level set equation (4). It has the important advantage of allowing - when applicable - to greatly reduce the computational effort required for solving the front tracking problem by solving a time-independent problem instead of a timedependent one. 


\section{Multidimensional time-fractional diffusion equation}

170 PDF of the interface particle displacement $p_{d}(\mathbf{x} ; t)$, which appears in Eq. (3), is assumed to be governed by the $d$-dimensional time-fractional diffusion equation. The latter is obtained from the classical diffusion equation by replacing the first order time derivative with a real order derivative operator $[36,37,38,39,20,40]$.

175 This replacement can be done by using the time fractional derivative operator in the Caputo sense or in the Riemann-Liouville sense. However, these two formulations are equivalent if standard initial condition is used [41]. Recently, in place of this operator replacement, the emergence of fractional kinetics in complex media has been explained within the standard Gaussian diffusion framework by the randomization due to the complexity of the environment of certain characteristic parameters of the medium [7].

Let $0<\beta \leqslant 1 / 2$ be a real parameter, then the time-fractional diffusion equation in the Caputo sense reads

$$
{ }_{*} D_{t}^{2 \beta} p_{d}=\nu \nabla^{2} p_{d}, \quad p_{d}(\mathbf{x} ; 0)=\delta(\mathbf{x}),
$$

where the coefficient $\nu$ is a positive constant with dimensions $[\nu]=[L]^{2}[T]^{-2 \beta}$ and ${ }_{*} D_{t}^{\mu}$ is the Caputo fractional derivative that is defined by the Laplace transformation

$$
\int_{0}^{+\infty} \mathrm{e}^{-s t}\left\{{ }_{*} D_{t}^{\mu} f(t)\right\} d t=s^{\mu} \tilde{f}(s)-\sum_{k=0}^{m-1} s^{\mu-1-k} f^{(k)}\left(0^{+}\right),
$$

with $m-1<\mu \leqslant m$ and $m \in \mathbb{N}$, where $f(t)$ is a sufficiently well-behaved function and $\widetilde{f}(s)$ its Laplace transform.

The time-fractional diffusion equation in the Riemann-Liouville sense reads

$$
\frac{\partial p_{d}}{\partial t}=\nu D_{t}^{1-2 \beta} \nabla^{2} p_{d}, \quad p_{d}(\mathbf{x} ; 0)=\delta(\mathbf{x})
$$

where $D_{t}^{\mu}$ is the Riemann-Liouville fractional derivative defined as

$$
\int_{0}^{+\infty} \mathrm{e}^{-s t}\left\{D_{t}^{\mu} f(t)\right\} d t=s^{\mu} \tilde{f}(s)
$$


provided that all the limiting values $f^{(k)}\left(0^{+}\right)$are finite with $k \in \mathbb{N}$ such that $0 \leqslant k \leqslant m-1$ where $m \in \mathbb{N}$ and $m-1<\mu \leqslant m$.

When $\beta=1 / 2$, both equations (6) and (7) reduce to the classical diffusion equation

$$
\frac{\partial p_{d}}{\partial t}=\nu \nabla^{2} p_{d}, \quad p_{d}(\mathbf{x} ; 0)=\delta(\mathbf{x})
$$

Applying Laplace and Fourier transformations to Eq. (6) as well as to Eq. (7), the fundamental solution $p_{d}(\mathbf{x} ; t)$ for an arbitrary spatial dimension $d$ results to be:

$$
p_{d}(\mathbf{x} ; t)=\frac{1}{(2 \pi)^{d+1} i} \int_{-i \infty}^{+i \infty}\left\{\int_{R^{d}} \frac{s^{2 \beta-1}}{s^{2 \beta}+\nu|\mathbf{k}|^{2}} \mathrm{e}^{i \mathbf{k} \cdot \mathbf{x}} d \mathbf{k}\right\} \mathrm{e}^{s t} d s
$$

which can be written as [20]:

$$
p_{d}(\mathbf{x} ; t)=\frac{1}{(2 \pi \sqrt{\nu})^{d / 2+1} i} \int_{-i \infty}^{+i \infty} s^{\beta(d / 2+1)-1} \mathrm{e}^{s t} \mathcal{K}_{d / 2-1}\left(\frac{s^{\beta}}{\sqrt{\nu}}|\mathbf{x}|\right) d s
$$

where $\mathcal{K}_{\theta}$ is the Macdonald function, or modified Bessel function of the second kind, of order $\theta$. By setting $s=-i \xi, \xi \in \mathbb{R}$, it follows that $s^{\beta}=\mathrm{e}^{-i \pi \beta \operatorname{sgn} \xi / 2}|\xi|^{\beta}$ and $p_{d}(\mathbf{x} ; t)$ is real [20]. However, integral (9) is not straightforwardly evaluable and a less computationally demanding formulation is desirable. It is shown in the following that such an alternative exists, since $p_{d}(\mathbf{x} ; t)$ can be written in terms of the $d$-dimensional $\mathbb{M}$-Wright/Mainardi function of order $\beta$ here denoted by $\mathbb{M}_{\beta}^{d}(\mathbf{x} ; t)$.

Let us start by reminding the following integral representation of the onedimensional $\mathcal{M}$-Wright/Mainardi function [21]:

$\mathbb{M}_{\eta / 2}^{1}(x ; t)=\frac{1}{t^{\eta / 2}} \mathcal{M}_{\eta / 2}\left(\frac{x}{t^{\eta / 2}}\right)=2 \int_{0}^{\infty} \frac{\mathrm{e}^{-x^{2} /(4 \tau)}}{\sqrt{4 \pi \tau}} \mathcal{M}_{\eta}\left(\frac{\tau}{t^{\eta}}\right) \frac{d \tau}{t^{\eta}}, \quad 0<\eta<1, x \in \mathbb{R}$

where the $\mathcal{M}$-Wright/Mainardi function $\mathcal{M}_{\eta}(z)$ is represented by series as $(z \in$ C) $[37,38,16,17]$

$$
\mathcal{M}_{\eta}(z)=\sum_{n=0}^{\infty} \frac{(-z)^{n}}{n ! \Gamma(-\eta n+(1-\nu))}=\frac{1}{\pi} \sum_{n=1}^{\infty} \frac{(-z)^{n-1}}{(n-1) !} \Gamma(\eta n) \sin (\pi \eta n) .
$$


Eq. (10) can be extended to a general spatial dimension $d$ by the extension of the Gaussian function as follows $\left(0<\eta \leqslant 1, \mathbf{x} \in \mathbb{R}^{d}\right)$

$$
\begin{aligned}
\mathbb{M}_{\eta / 2}^{d}(\mathbf{x} ; t) & =2 \int_{0}^{+\infty} \frac{\mathrm{e}^{-|\mathbf{x}|^{2} /(4 \tau)}}{(4 \pi \tau)^{d / 2}} \mathcal{M}_{\eta}\left(\frac{\tau}{t^{\eta}}\right) \frac{d \tau}{t^{\eta}} \\
& =2 \int_{0}^{+\infty} \prod_{i=1}^{d} \frac{\mathrm{e}^{-x_{i}^{2} /(4 \tau)}}{(4 \pi \tau)^{1 / 2}} \mathcal{M}_{\eta}\left(\frac{\tau}{t^{\eta}}\right) \frac{d \tau}{t^{\eta}} .
\end{aligned}
$$

When $d=1$, Eq. (11) coincides with Eq. (10). Since the following Laplace transform pair holds [39]:

$$
\int_{0}^{+\infty} \mathrm{e}^{-s t} \mathcal{M}_{\eta}\left(\frac{\tau}{t^{\eta}}\right) \frac{d t}{t^{\eta}}=s^{\eta-1} \mathrm{e}^{-\tau s^{\eta}}
$$

after the application of Laplace and Fourier transformations to (11) it follows that

$$
\begin{aligned}
\mathbb{M}_{\eta / 2}^{d}(\mathbf{x} ; t) & =\frac{2}{(2 \pi)^{d+1} i} \int_{-i \infty}^{+i \infty}\left\{\int_{\mathbb{R}^{d}}\left[\int_{0}^{+\infty} \prod_{i=1}^{d} \mathrm{e}^{-\tau k_{i}^{2}} \mathrm{e}^{-\tau s^{\eta}} d \tau\right] \mathrm{e}^{i \mathbf{k} \cdot \mathbf{x}} d \mathbf{k}\right\} s^{\eta-1} \mathrm{e}^{s t} d s \\
& =\frac{2}{(2 \pi)^{d+1} i} \int_{-i \infty}^{+i \infty}\left\{\int_{\mathbb{R}^{d}}\left[\int_{0}^{+\infty} \mathrm{e}^{-\tau\left(s^{\eta}+\nu k^{2}\right)} d \tau\right] \mathrm{e}^{i \mathbf{k} \cdot \mathbf{x}} d \mathbf{k}\right\} s^{\eta-1} \mathrm{e}^{s t} d s \\
& =\frac{2}{(2 \pi)^{d+1} i} \int_{-i \infty}^{+i \infty}\left\{\int_{\mathbb{R}^{d}} \frac{s^{\eta-1}}{s^{\eta}+k^{2}} \mathrm{e}^{i \mathbf{k} \cdot \mathbf{x}} d \mathbf{k}\right\} \mathrm{e}^{s t} d s,
\end{aligned}
$$

The latter, together with (8) and making use of the change of variables $k \rightarrow \sqrt{\nu} k$ and $\mathbf{x} \rightarrow \mathbf{x} / \sqrt{\nu}$, allows to conclude that

$$
p_{d}(\mathbf{x} ; t)=\frac{1}{2 \sqrt{\nu}} \mathbb{M}_{\beta}^{d}\left(\frac{\mathbf{x}}{\sqrt{\nu}} ; t\right)=\int_{0}^{+\infty} \frac{\mathrm{e}^{-|\mathbf{x}|^{2} /(4 \nu \tau)}}{(4 \pi \nu \tau)^{d / 2}} \mathcal{M}_{2 \beta}\left(\frac{\tau}{t^{2 \beta}}\right) \frac{d \tau}{t^{2 \beta}} .
$$

Eq. (12) is both analytically and numerically less cumbersome than (8), thus providing a computationally attractive alternative to Eq. (9). Comparing $p_{1}$ and $p_{3}$ obtained by means of Eq. (12), it holds

$$
p_{3}(\mathbf{x} ; t)=-\frac{1}{2 \pi r} \frac{\partial}{\partial r} p_{1}(r ; t), \quad r=|\mathbf{x}|,
$$

which provides a well-known relation between the one-dimensional and threedimensional PDFs $p_{1}$ and $p_{3}[20]$. 
It is noted that the previously mentioned constraint on parameter $\beta(0<\beta \leqslant$ $1 / 2$ ) guarantees to have a proper diffusion process characterized by an unimodal $\operatorname{PDF}[37,39]$. In fact, when $1 / 2<\beta<1$ function $\mathcal{M}_{\beta}(z)$ is bimodal and no diffusion process is modelled by $p_{3}$, because the latter assumes also negative values, see (13). The subdiffusive character of diffusion is highlighted by the particle variance $\sigma_{d}^{2}$ which in one-, two- and three-dimensions $(d=1,2,3)$ is $[39,20]$ :

$$
\sigma_{1}^{2}=\int_{-\infty}^{+\infty} x^{2} p_{1}(x ; t) d x=\frac{2 \nu t^{2 \beta}}{\Gamma(2 \beta+1)}, \quad \sigma_{2}^{2}=2 \sigma_{1}^{2}, \quad \sigma_{3}^{2}=3 \sigma_{1}^{2} .
$$

In Figure 1, the fundamental solutions of time-fractional diffusion equation (7) are presented for the one-, two- and three-dimensional case and for five different values of parameter $\beta$, i.e. $\beta=0.1,0.2,0.3,0.4$ and 0.5 , corresponding the latter to the case of ordinary diffusion and the other values to subdiffusive processes.

Fundamental solutions have been numerically calculated on the basis of the analytical representation given in Eq. (12). In particular the numerical evaluation of the $\mathcal{M}$-Wright/Mainardi function $\mathcal{M}_{\eta}(z)$ and of the fundamental solutions in $d$ dimensions $p_{d}(\mathbf{x} ; t)$ have been performed by means of a python library (pyMlib) based on state-of-the-art algorithms for the Mittag-Leffler function [42] as well as on standard Laplace inversion and standard integration techniques available in the Integrate submodule of the SciPy scientific library [43].

In this respect, it is here reminded that the Laplace transform of the $\mathcal{M}$ Wright/Mainardi function is $[39,16,17]$

$$
\int_{0}^{+\infty} \mathrm{e}^{-s t} \mathcal{M}_{\eta}(t) d t=E_{\eta}(-s), \quad 0<\eta<1, \quad \operatorname{Re}(s)>0,
$$

where $E_{\eta}(z)$ is the Mittag-Leffler function defined by [44]

$$
E_{\rho}(z)=\sum_{n=0}^{\infty} \frac{z^{n}}{\Gamma(\rho n+1)}, \quad \rho>0, \quad z \in \mathbb{C} .
$$


(a)

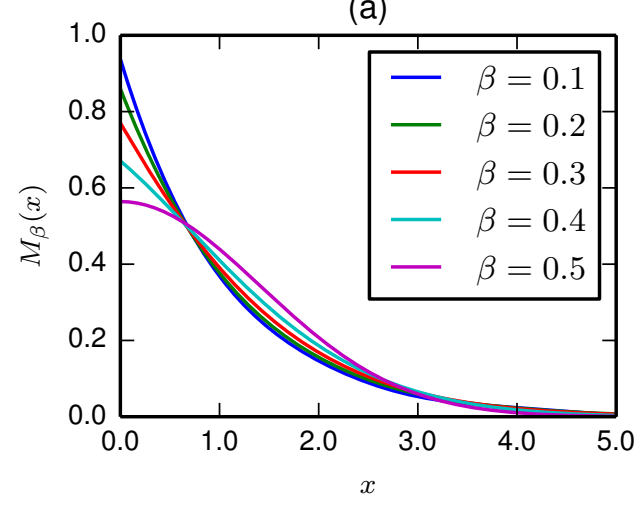

(b)

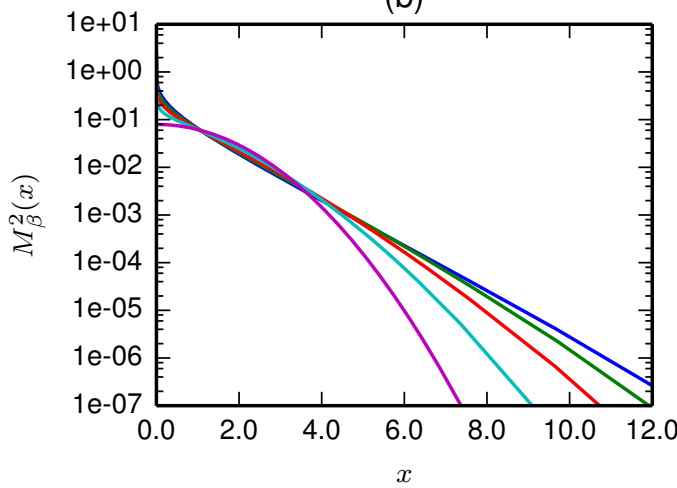

(c)

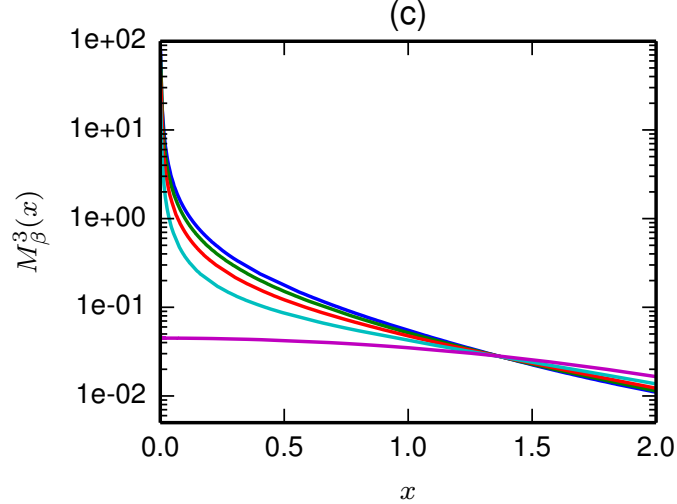

Figure 1: Fundamental solutions of the time-fractional diffusion equation (7) in the (a) onedimensional, (b) two-dimensional and (c) three-dimensional cases for five values of the parameter $\beta: \beta=0.1,0.2,0.3,0.4$ and 0.5 . 


\section{Numerical results}

In this Section the profiles of the effective indicator $\varphi_{e}(\mathbf{x}, t)$ defined in (3) are shown and discussed. In particular, to compute (3) the indicator function $\mathcal{I}_{\Omega}(\mathbf{x}, t)$ is obtained according to definition (1) by solving the level set equation (4) with constant velocity $\mathcal{V}$, and the PDF $p_{d}(\mathbf{x} ; t \mid \overline{\mathbf{x}})=p_{d}(\mathbf{x}-\overline{\mathbf{x}} ; t)$ is the fundamental solution of the $d$-dimensional time-fractional diffusion given in (12). The algorithm used to compute (12) is described in Section 3.

The evolution of random fronts propagating in subdiffusive and diffusive media has been numerically investigated by means of a software package developed for this goal. The code makes use of a general-purpose library (liblsm90) which aims at providing a robust and efficient tool for studying the evolution of co-dimension one fronts propagating in one-, two- and three-dimensional system. The library, written in Fortran2008/OpenMP, is presently still under active development and is intended to be released as open source software under the GNU/GPLv3 licence. Along with standard algorithms useful for the calcu225 lation of the front evolution by means of the classical LSM, the library includes Fast Marching Method (FMM) algorithms, which allow to efficiently solve the eikonal equation (5) arising from the classical LSM formulation in place of the standard Hamilton-Jacobi equation whenever it is possible, as in the case here under consideration.

All the results presented in the following have been obtained making use of the above-mentioned software and have been post-processed for exposition purposes by means of open source software such as SciPy [43] and Matplotlib [45] in the IPython framework [46].

All the calculations have been performed by means of the computational server available at the Basque Center for Applied Mathematics (BCAM) in Bilbao, Basque Country - Spain.

A selection of the results concerning the propagation of random fronts in media characterized by anomalous diffusion is presented in this Section. The 
discussion is restricted to fronts propagating in two-dimensional space, the onedimensional case being discussed elsewhere [8] and a thorough analysis including three-dimensional cases being still under development.

Once the main features of one-dimensional propagation of plane fronts have been investigated [8], the two-dimensional case has the relevant advantage of allowing to investigate practical situations of effective interest in which multidimensional effects possibly come into play, still avoiding the complexity of a three-dimensional analysis.

The analysis proposed in this section concerns the investigation of the propagation of random fronts in two-dimensional subdiffusive and diffusive media, focusing on the effects of the diffusion coefficient $\nu$, the parameter $\beta$ and the level set velocity $\mathcal{V}$.

First the case of an initial front profile with circular shape is discussed, to focus on the effects of $\nu, \beta$ and $\mathcal{V}$ avoiding the two-dimensional effects connected to a non-axisymmetric initial front profile. These effects are then discussed in the case of a square-shaped initial front profile, with the main aim of investigating the effects of the parameter $\beta$ on the short-term and long-term evolution of the random front.

In both cases, the computational domain is the rectangular region $[-2 \mathrm{~m}, 2 \mathrm{~m}] \times$ [ $-2 \mathrm{~m}, 2 \mathrm{~m}]$. The circular profile has a radius $r=0.4 \mathrm{~m}$, and the square-shaped profile has a side $\ell$ such that in both cases the region enclosed by the front when ${ }_{260} t=0 \mathrm{~s}$ has the same area (i.e. $\ell \approx 0.7 \mathrm{~m}$ ). The center of both regions is located in $(x, y)=(0,0)$.

In the scenario involving a circular initial front profile, when the diffusion coefficient $\nu$ is small enough, it is seen that for any value under investigation of the parameter $\beta$ the only outstanding feature of the propagating front is its smoothing with respect to the sharp front that characterizes the deterministic case, i.e. a non-diffusive system. Profiles representative of this behaviour are shown in Fig. 2, where the numerical computations of $\varphi_{e}(\mathbf{x}, t)$ as defined in (3) are plotted together with the deterministic front (dashed line) at different 
time instants for the case $\nu=10^{-3} \mathrm{~m}^{2} \mathrm{~s}^{-2 \beta}$ and two different values of the above is less relevant for the case with larger $\mathcal{V}$.

This behaviour suggests a balance between the diffusion coefficient $\nu$ and the 
(a) $\mathcal{V}=0.1 \mathrm{~m} / \mathrm{s}, \mathrm{t}=0.5 \mathrm{~s}$

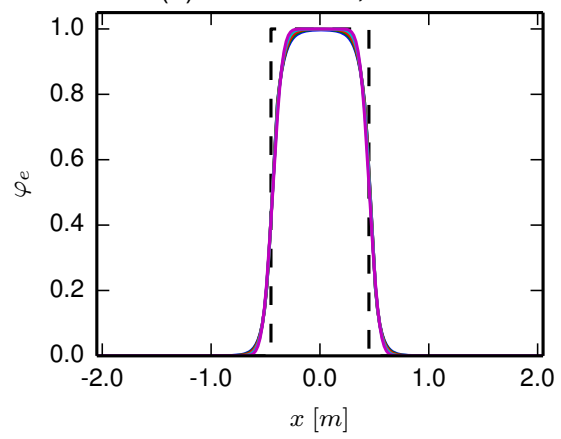

(c) $\mathcal{V}=0.1 \mathrm{~m} / \mathrm{s}, \mathrm{t}=3.0 \mathrm{~s}$

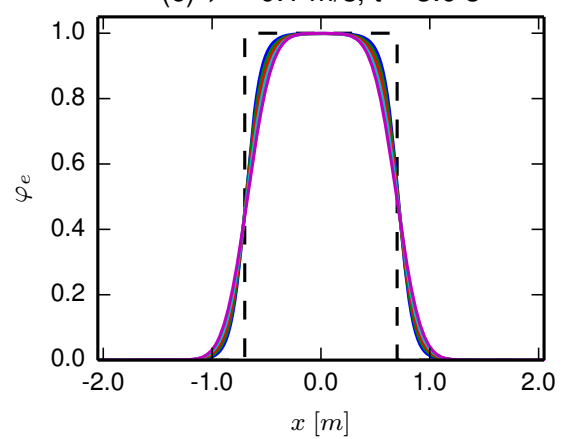

(e) $\mathcal{V}=0.1 \mathrm{~m} / \mathrm{s}, \mathrm{t}=10.0 \mathrm{~s}$

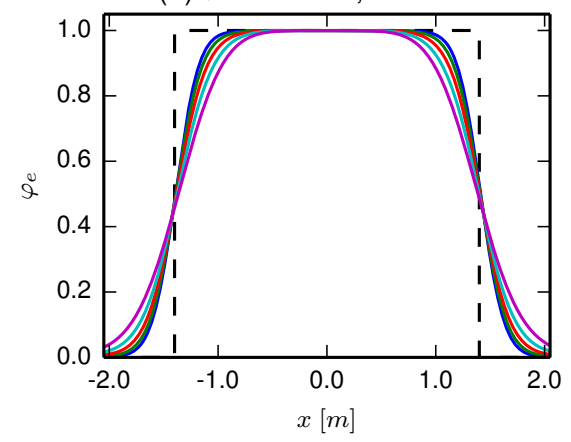

(b) $\mathcal{V}=1.0 \mathrm{~m} / \mathrm{s}, \mathrm{t}=0.05 \mathrm{~s}$

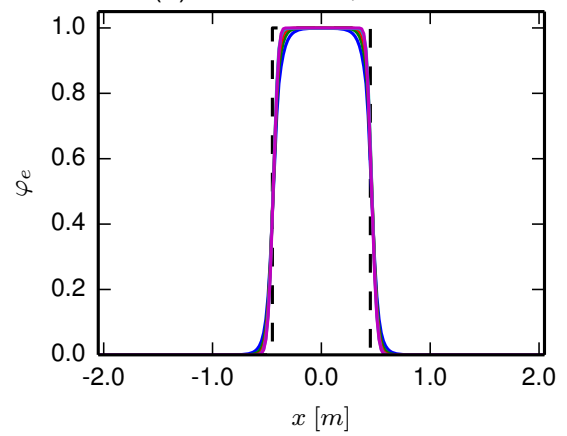

(d) $\mathcal{V}=1.0 \mathrm{~m} / \mathrm{s}, \mathrm{t}=0.3 \mathrm{~s}$

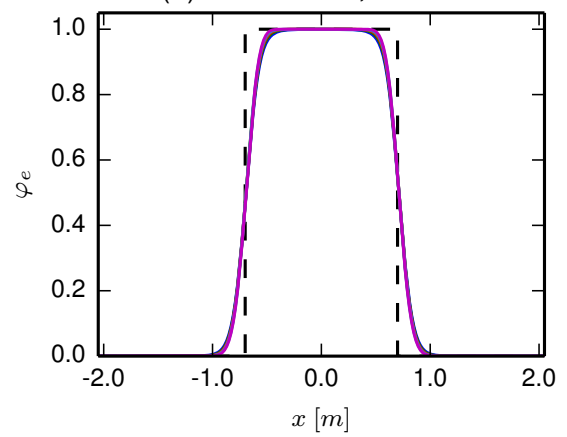

(f) $\mathcal{V}=1.0 \mathrm{~m} / \mathrm{s}, \mathrm{t}=1.0 \mathrm{~s}$

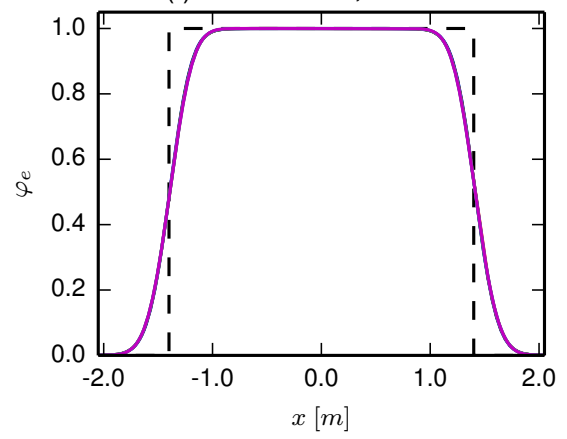

Figure 2: Cross-section at $y=0$ of the $\varphi_{e}$ fields corresponding to five values of the parameter $\beta(\beta=0.1,0.2,0.3,0.4,0.5)$ on the two-dimensional domain $[-2 \mathrm{~m}, 2 \mathrm{~m}] \times[-2 \mathrm{~m}, 2 \mathrm{~m}]$ at three values of the time $t$ (top, middle and bottom row) and for two values of velocity $\mathcal{V}$ (left and right column). The initial profile at $t=0 \mathrm{~s}$ is circular, with centre $C=(0,0)$ and radius $r=0.4 \mathrm{~m}$. The diffusion coefficient is $\nu=0.001 \mathrm{~m}^{2} \mathrm{~s}^{-2 \beta}$. 

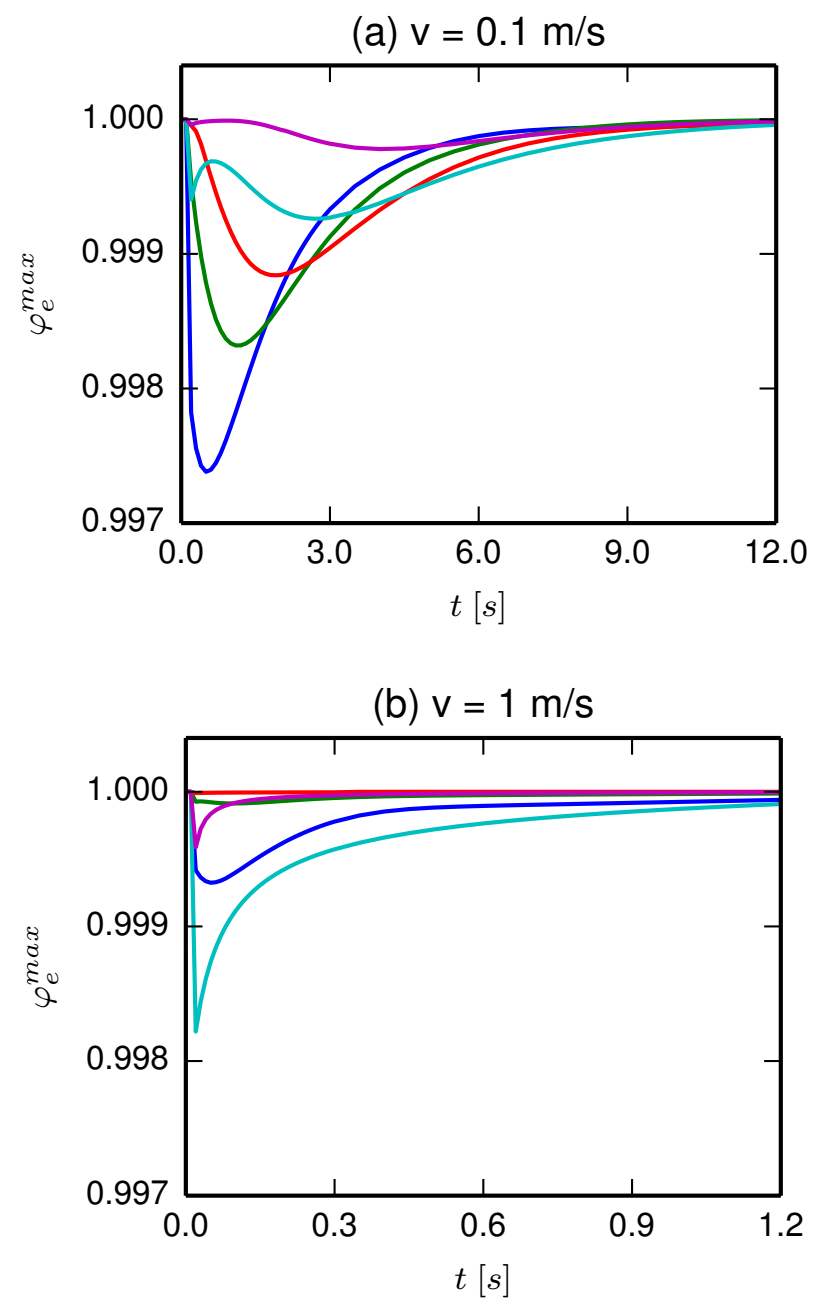

Figure 3: Time evolution of the maximum value of $\varphi_{e}\left(\varphi_{e}^{\max }\right)$ corresponding to five values of the parameter $\beta$ ( $\beta=0.1,0.2,0.3,0.4,0.5)$ for the case discussed in Figure 2. 
velocity $\mathcal{V}$ from which the timescale $\tau_{w}$ of this weakening effect can be estimated. In particular, such timescale emerges to be defined as

$$
\tau_{w}=\frac{\sqrt{\nu \tau_{u}^{2 \beta}}}{\mathcal{V}},
$$

where $\tau_{u}$ is the unit of measurement for the temporal variable $t$ and then it can be stated equal to 1 . The estimation of the timescale $\tau_{w}$ for the weakening process is largely dependent on the parameter $\beta$, as expected, as well as on the velocity $\mathcal{V}$. In fact, the comparison of Fig. 5(a) and Fig. 5(b) shows how subdiffusive processes with small values of $\beta$ may be responsible for smaller weakening effect with respect to subdiffusive processes with larger values of $\beta$ 315 (and also with respect to normal diffusive processes) when small values of the velocity $\mathcal{V}$ are involved. Interestingly, the situation can be reversed when larger values of $\mathcal{V}$ are involved.

The features of the random fronts described so far for the case with $\nu=$ $0.01 \mathrm{~m}^{2} \mathrm{~s}^{-2 \beta}$ remains qualitatively unchanged as the diffusion coefficient $\nu$ increases, but an important remark is in order. Depending on the physical interpretation of the indicator $\varphi_{e}$, it may - or may not - be meaningful to introduce the concept of "quencing" of the propagating front. In fact, assuming that the front is located, say, at the position for which the effective indicator $\varphi_{e}$ reaches 
(a) $\mathcal{V}=0.1 \mathrm{~m} / \mathrm{s}, \mathrm{t}=0.5 \mathrm{~s}$

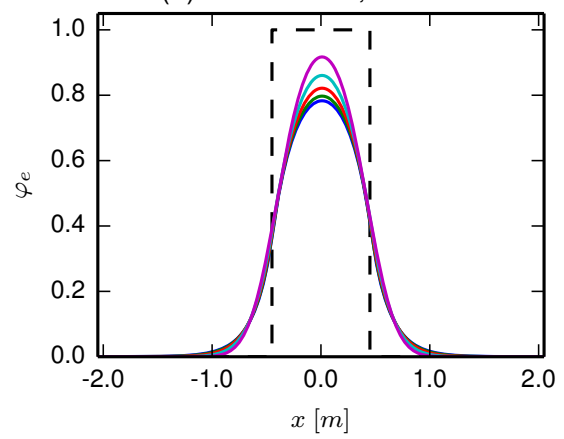

(c) $\mathcal{V}=0.1 \mathrm{~m} / \mathrm{s}, \mathrm{t}=3.0 \mathrm{~s}$

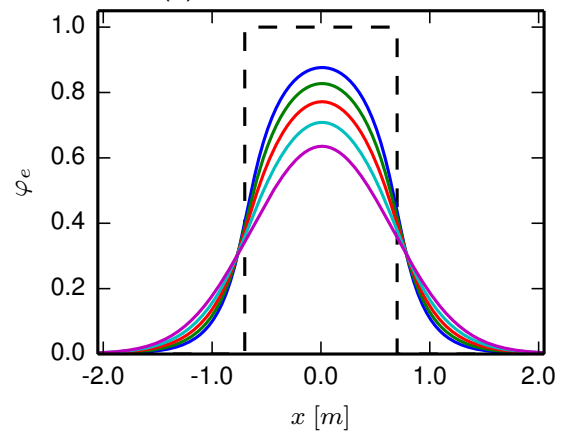

(e) $\mathcal{V}=0.1 \mathrm{~m} / \mathrm{s}, \mathrm{t}=10.0 \mathrm{~s}$

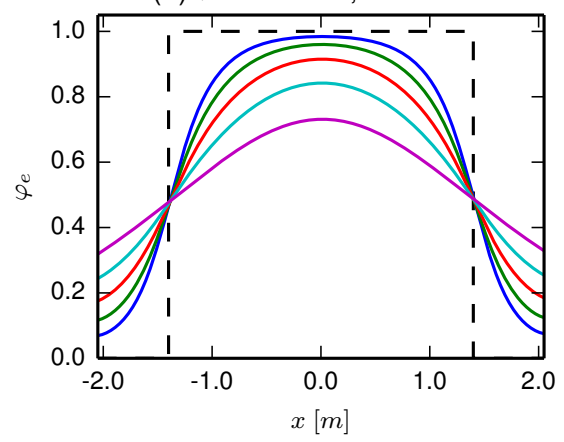

(b) $\mathcal{V}=1.0 \mathrm{~m} / \mathrm{s}, \mathrm{t}=0.05 \mathrm{~s}$

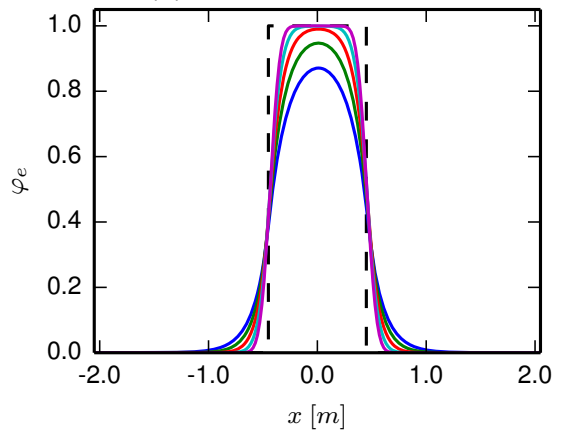

(d) $\mathcal{V}=1.0 \mathrm{~m} / \mathrm{s}, \mathrm{t}=0.3 \mathrm{~s}$

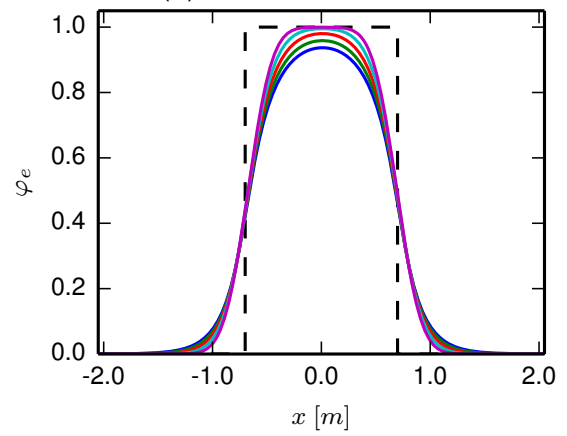

(f) $\mathcal{V}=1.0 \mathrm{~m} / \mathrm{s}, \mathrm{t}=1.0 \mathrm{~s}$

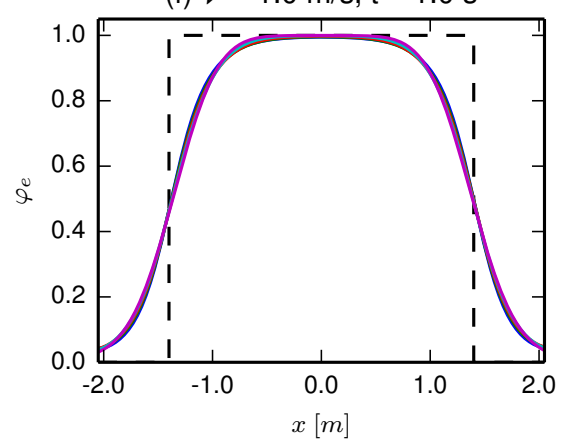

Figure 4: The same as in Figure 2 but with $\nu=0.01 \mathrm{~m}^{2} \mathrm{~s}^{-2 \beta}$. 
(a) $v=0.1 \mathrm{~m} / \mathrm{s}$

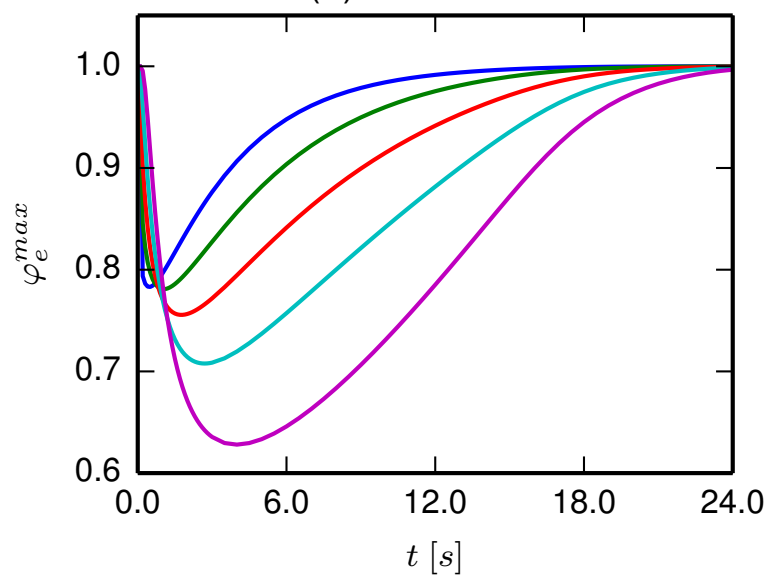

(b) $v=1 \mathrm{~m} / \mathrm{s}$

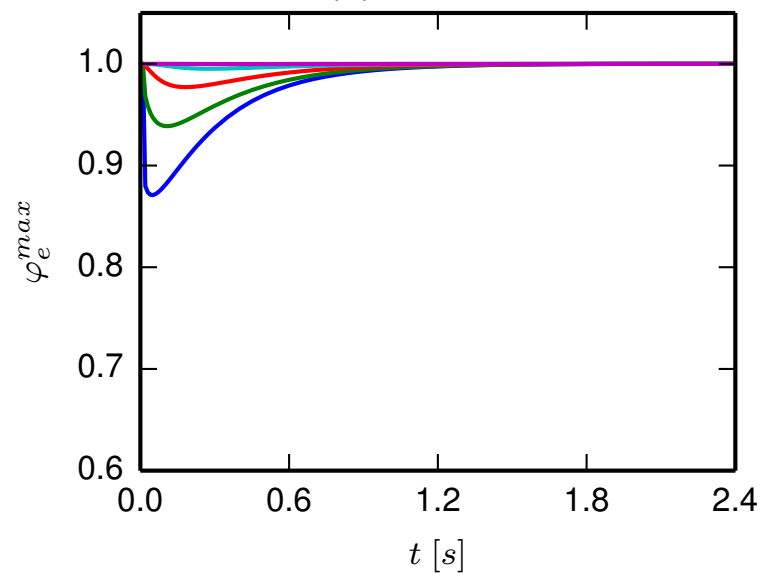

Figure 5: Time evolution of the maximum value of $\varphi_{e}\left(\varphi_{e}^{\max }\right)$ corresponding to five values of the parameter $\beta$ ( $\beta=0.1,0.2,0.3,0.4,0.5)$ for the case discussed in Figure 4. 
the threshold value $\varphi_{e}^{\text {th }}$, it might be meaningful to argue that when the effective because, when $t<\tau_{w}$, the smaller the parameter $\beta$ the larger the particle variance (14). Among the three cases shown in Fig. 8, Fig. 9 and Fig. 10, the case 
(a) $\mathcal{V}=0.1 \mathrm{~m} / \mathrm{s}, \mathrm{t}=0.5 \mathrm{~s}$

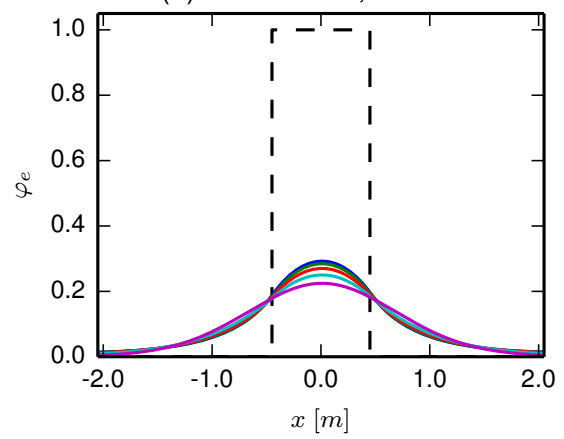

(c) $\mathcal{V}=0.1 \mathrm{~m} / \mathrm{s}, \mathrm{t}=3.0 \mathrm{~s}$

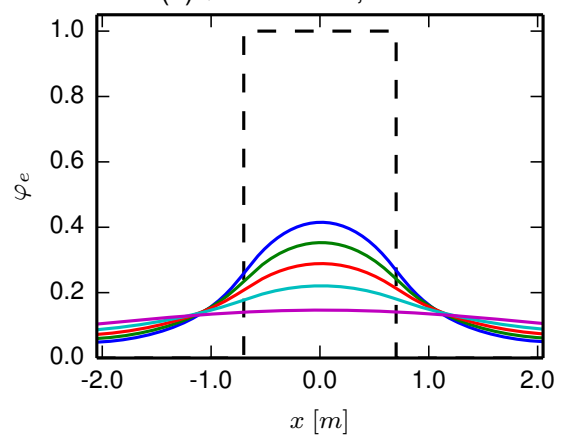

(e) $\mathcal{V}=0.1 \mathrm{~m} / \mathrm{s}, \mathrm{t}=10.0 \mathrm{~s}$

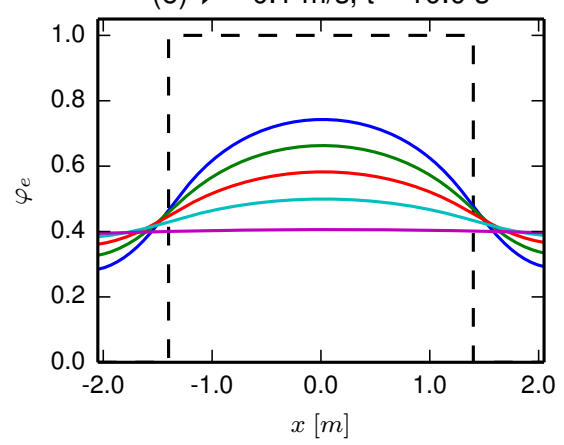

(b) $\mathcal{V}=1.0 \mathrm{~m} / \mathrm{s}, \mathrm{t}=0.05 \mathrm{~s}$

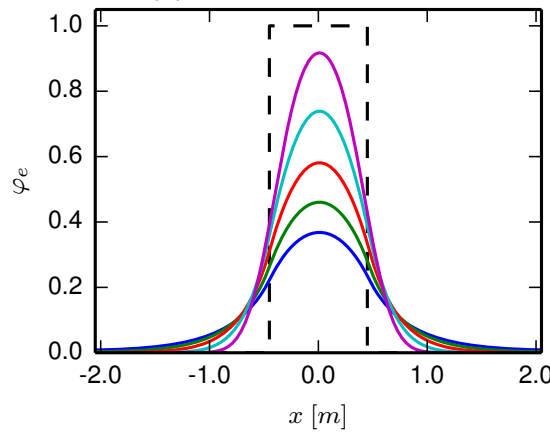

(d) $\mathcal{V}=1.0 \mathrm{~m} / \mathrm{s}, \mathrm{t}=0.3 \mathrm{~s}$

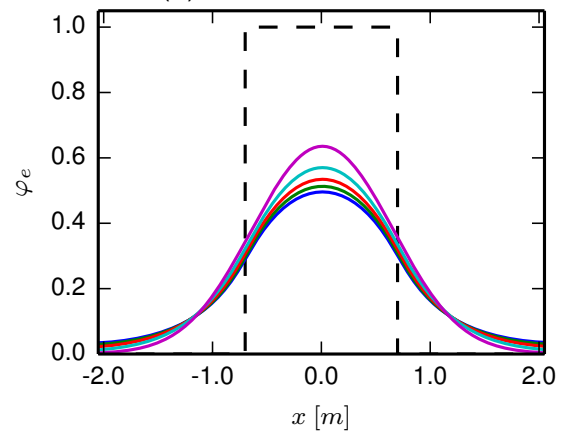

(f) $\mathcal{V}=1.0 \mathrm{~m} / \mathrm{s}, \mathrm{t}=1.0 \mathrm{~s}$

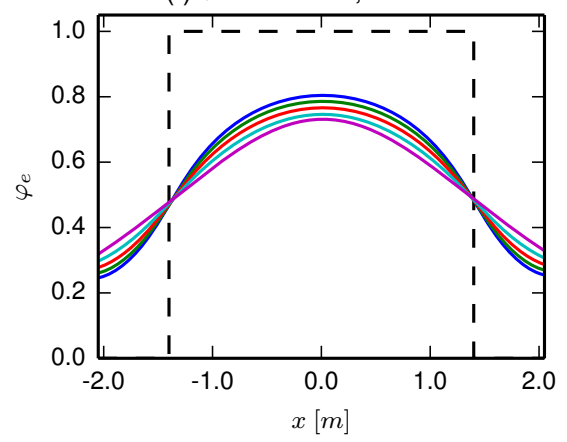

Figure 6: The same as in Figure 2 but with $\nu=0.1 \mathrm{~m}^{2} \mathrm{~s}^{-2 \beta}$. 
(a) $v=0.1 \mathrm{~m} / \mathrm{s}$

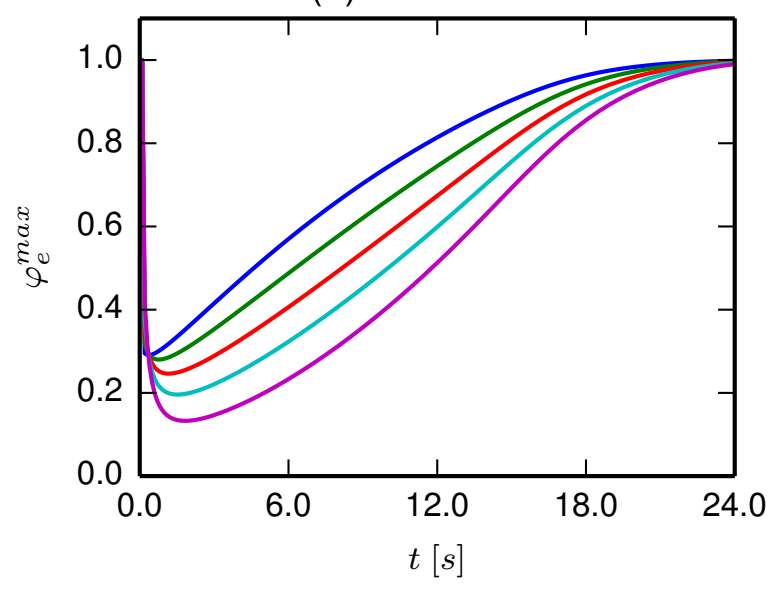

(b) $v=1 \mathrm{~m} / \mathrm{s}$

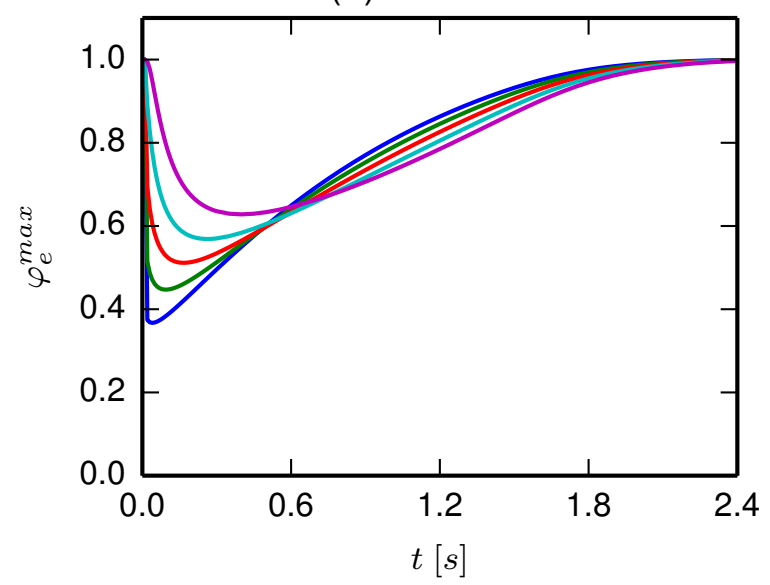

Figure 7: Time evolution of the maximum value of $\varphi_{e}\left(\varphi_{e}^{\max }\right)$ corresponding to five values of the parameter $\beta$ ( $\beta=0.1,0.2,0.3,0.4,0.5)$ for the case discussed in Figure 6 . 

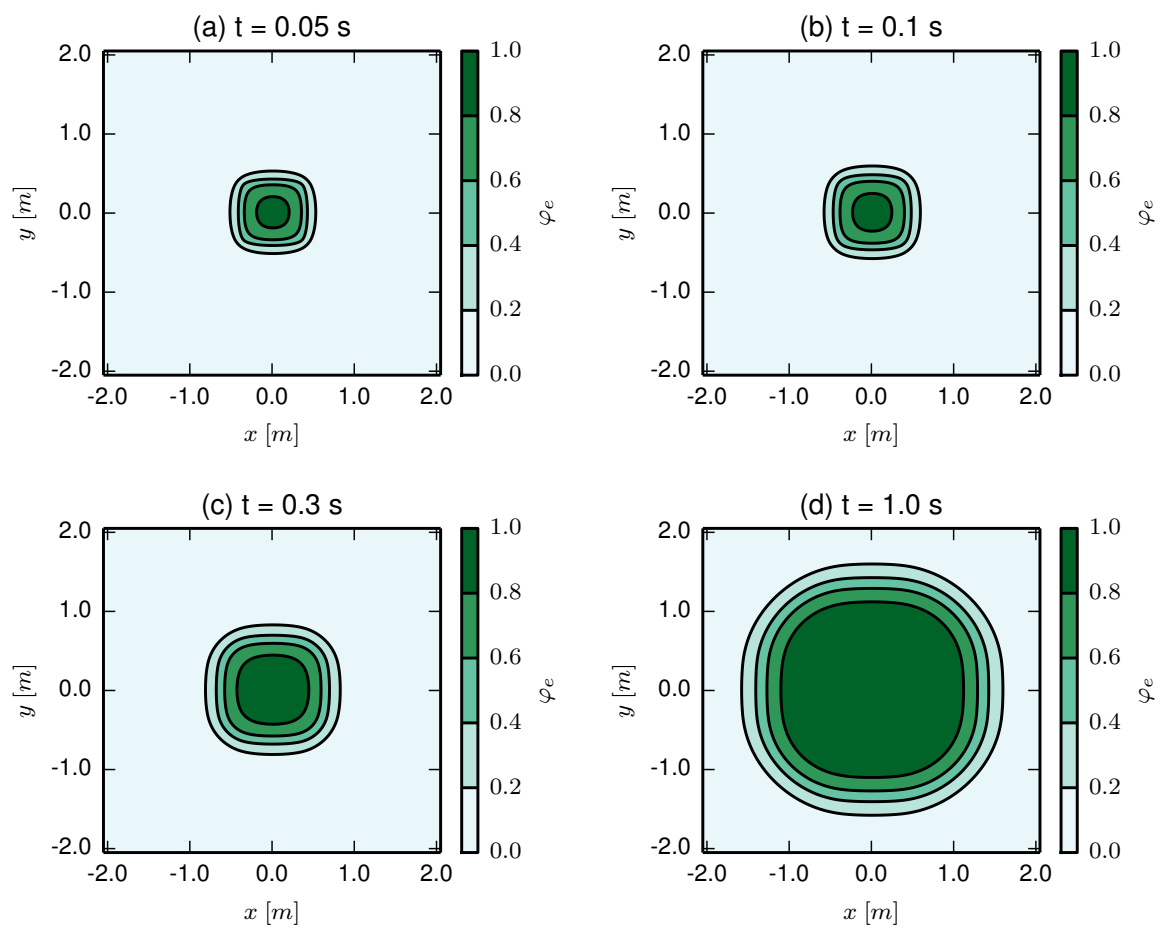

Figure 8: Distribution of the $\varphi_{e}$ field for the case with $\beta=0.1$ on the two-dimensional domain $[-2 \mathrm{~m}, 2 \mathrm{~m}] \times[-2 \mathrm{~m}, 2 \mathrm{~m}]$ at four instant times. The initial profile at $t=0 \mathrm{~s}$ is a square, with center $C=(0,0)$ and side $\ell=0.7 \mathrm{~m}$. The diffusion coefficient is $\nu=0.01 \mathrm{~m}^{2} \mathrm{~s}^{-2 \beta}$ and $\mathcal{V}=1 \mathrm{~ms}^{-1}$.

characterized by the ordinary diffusion process is clearly the one in which the initial front profile remain almost unchanged for a longer time, being the profile also sharper. For large times - as seen in Fig. 8(d), Fig. 9(d) and Fig. 10(d) - the diffusion process leads to similar front profiles in all the discussed cases, being the effective indicator field $\varphi_{e}$ undistinguishable, as a matter of fact, in all the three cases.

\section{Conclusions}

In this paper, a multi-dimensional model to study the evolution of random fronts in media in which anomalous diffusion takes place has been presented. 
(a) $t=0.05 \mathrm{~s}$

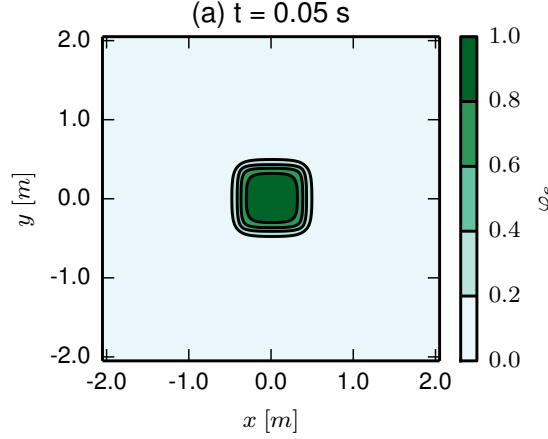

(c) $t=0.3 \mathrm{~s}$

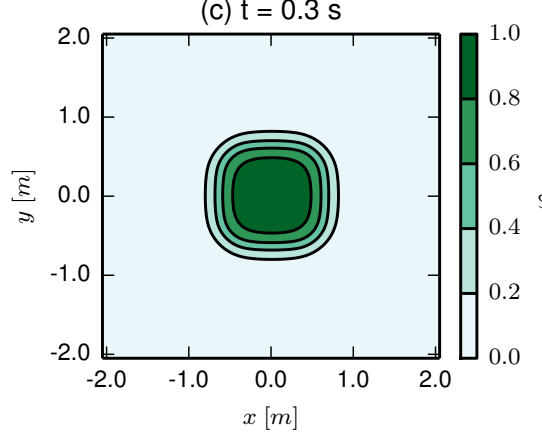

(b) $\mathrm{t}=0.1 \mathrm{~s}$

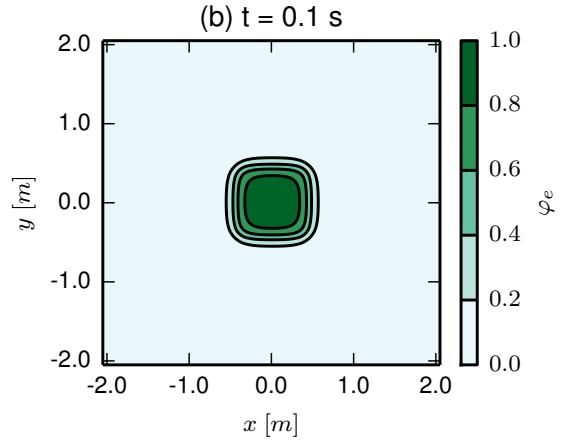

(d) $\mathrm{t}=1.0 \mathrm{~s}$

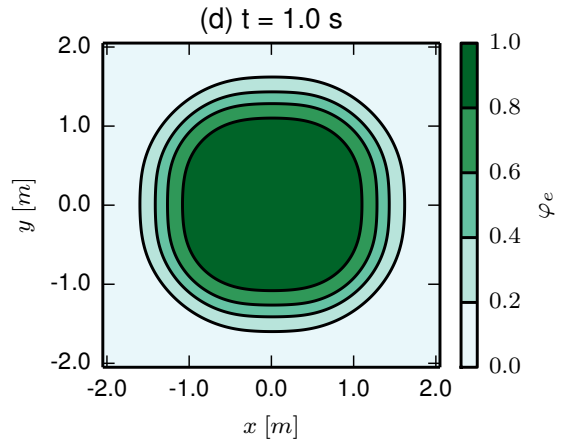

Figure 9: The same as in Figure 8 but for the case with $\beta=0.3$. 
(a) $t=0.05 \mathrm{~s}$

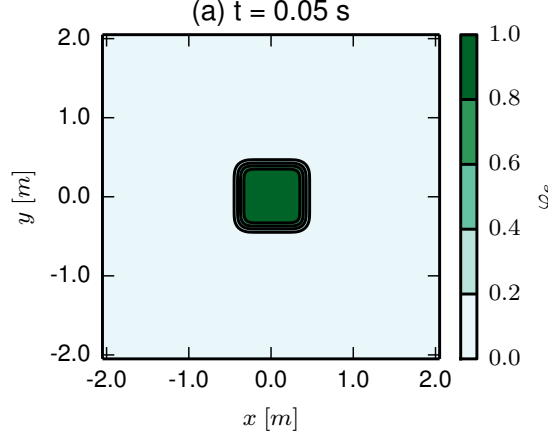

(c) $t=0.3 \mathrm{~s}$

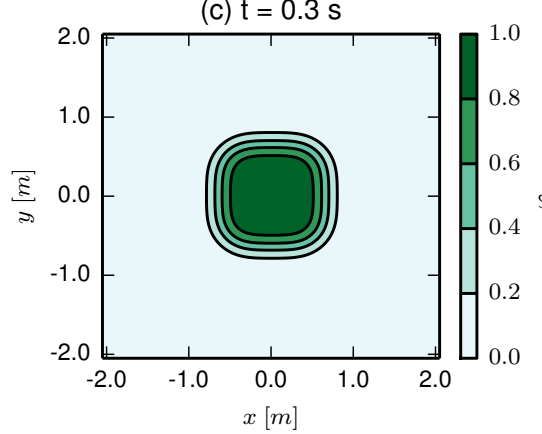

(b) $\mathrm{t}=0.1 \mathrm{~s}$

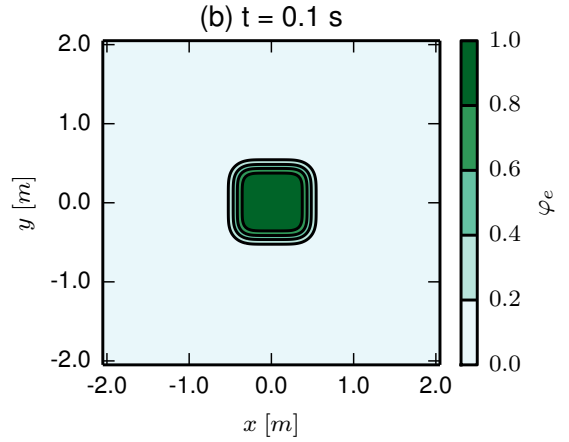

(d) $\mathrm{t}=1.0 \mathrm{~s}$

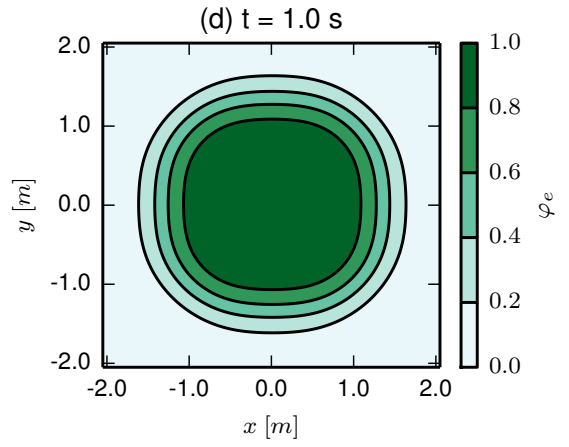

Figure 10: The same as in Figure 8 but for the case with $\beta=0.5$. 
In the proposed model, the position of the random front is obtained as the weighted mean of fronts calculated by means of the LSM, using as weightdiffusion (namely, the diffusion coefficient $\nu$ ) is small enough, the propagating front is smoothed-out with respect to the sharp front obtained in the deter- 
ministic, i.e. non-diffusive case, but the overall qualitative behaviour of the front propagation is not affected by the diffusive phenomena taking place in the medium. On contrast, when the intensity of the diffusion is large, the qualitative behaviour of the random fronts sensibly changes: in this case the smoothing of the front may be so significant as to even compromise the existence of the region enclosed by the propagating front (and the front itself, in turn). This effect, which has been referred to as a weakening effect of the diffusion phenomena on the region bounded by the propagating front can be, as shown, a transient effect gradually vanishing as the front propagates, or even a phenomenon which drastically affects the very existence of the front, depending on the intensity of the diffusion itself. In both cases, it has been shown how the velocity of propagation of the deterministic front plays a significant role in the features of the front propagation, as the above-discussed weakening effect is reduced as such velocity increases.

Moreover, aside from depending on the intensity of the diffusion phenomena through the diffusion coefficient $\nu$, the above-mentioned weakening effects has also been shown to be more pronounced as the subdiffusive nature of the diffusion increases. The timescale $\tau_{w}$ of this weakening process has been estimated.

As far as the analysis of the propagation of an initially square-shaped front is concerned, it has been shown that, in the case under investigation and for short elapsed times (i.e. $t<\tau_{w}$ ), small values of the parameter $\beta$ leads to a smearing of the initial front shape remarkably faster than in the case of ordinary diffusion. This occurs because, when $t<\tau_{w}$, the smaller the coefficient $\beta$ the larger the particle variance (14).

The LSM library utilized for the purpose of this investigation, featuring optimized FMM algorithms as well, is intended to be released as open source software under the GNU General Public License version 3 (GPLv3). 
This research is supported by GNFM/INdAM Young Researchers Project 2013, by Bizkaia Talent and European Commission through COFUND programme under Grant AYD-000-226, and also by the Basque Government through the BERC 2014-2017 program and by the Spanish Ministry of Economy and Competitiveness MINECO: BCAM Severo Ochoa accreditation SEV-2013-0323. GP would like to thank Prof. F. Mainardi for continuous support, suggestions and encouragement.

\section{References}

[1] E. Barkai, Y. Garini, R. Metzler, Strange kinetics of single molecules in living cells, Phys. Today 65 (8) (2012) 29-35.

[2] F. Höfling, T. Franosch, Anomalous transport in the crowded world of biological cells, Rep. Prog. Phys. 76 (2013) 046602.

[3] R. Metzler, J.-H. Jeon, A. G. Cherstvy, E. Barkai, Anomalous diffusion models and their properties: non-stationarity, non-ergodicity, and ageing at the centenary of single particle tracking, Phys. Chem. Chem. Phys. 16 (2014) 24128-24164.

[4] D. del Castillo-Negrete, Non-diffusive, non-local transport in fluids and plasmas, Nonlin. Processes Geophys. 17 (2010) 795-807.

[5] R. Metzler, J. Klafter, The restaurant at the end of the random walk: recent developments in fractional dynamics descriptions of anomalous dynamical processes, J. Phys. A: Math. Theor. 37 (31) (2004) R161-R208.

[6] A. Blumen, A. A. Gurtovenko, S. Jespersen, Anomalous diffusion and relaxation in macromolecular systems, J. Non-Cryst. Solids 305 (2002) 71-80.

[7] G. Pagnini, Short note on the emergence of fractional kinetics, Physica A 409 (2014) 29-34. 
[8] A. Mentrelli, G. Pagnini, Random front propagation in fractional diffusive systems, submitted.

[9] J. A. Sethian, P. Smereka, Level set methods for fluid interfaces, Ann. Rev. Fluid Mech. 35 (2003) 341-372.

450 [10] G. Pagnini, E. Bonomi, Lagrangian formulation of turbulent premixed combustion, Phys. Rev. Lett. 107 (2011) 044503.

[11] G. Pagnini, L. Massidda, Modelling turbulence effects in wildland fire propagation by the randomized level-set method, Tech. Rep 2012/PM12a, CRS4, revised Version August 2014. arXiv:1408.6129 (July 2012).

[12] G. Pagnini, L. Massidda, The randomized level-set method to model turbulence effects in wildland fire propagation, in: D. Spano, V. Bacciu, M. Salis, C. Sirca (Eds.), Modelling Fire Behaviour and Risk. Proceedings of the International Conference on Fire Behaviour and Risk. ICFBR 2011, Alghero, Italy, October 4-6 2011, 2012, pp. 126-131, ISBN 978-88-904409-7-7.

[13] G. Pagnini, A. Mentrelli, Modelling wildland fire propagation by tracking random fronts, Nat. Hazards Earth Syst. Sci. 14 (2014) 2249-2263.

[14] G. Pagnini, A model of wildland fire propagation including random effects by turbulence and fire spotting, in: Proceedings of XXIII Congreso de Ecuaciones Diferenciales y Aplicaciones XIII Congreso de Matemática Aplicada. Castelló, Spain, 9-13 September 2013, 2013, pp. 395-403.

[15] G. Pagnini, Fire spotting effects in wildland fire propagation, in: F. Casas, V. Martínez (Eds.), Advances in Differential Equations and Applications, Vol. 4 of SEMA SIMAI Springer Series, Springer International Publishing Switzerland, 2014, pp. 203-216, iSBN: 978-3-319-06952-4. (eBook: 978-3319-06953-1).

[16] F. Mainardi, A. Mura, G. Pagnini, The M-Wright function in timefractional diffusion processes: A tutorial survey, Int. J. Diff. Equations 2010 (2010) 104505. 
[17] F. Mainardi, Fractional Calculus and Waves in Linear Viscoelasticity, Imperial College Press, London, 2010.

[18] G. Pagnini, The M-Wright function as a generalization of the Gaussian density for fractional diffusion processes, Fract. Calc. Appl. Anal. 16 (2) (2013) 436-453.

[19] I. Podlubny, Fractional Differential Equations, Academic Press, San Diego, 1999.

[20] A. Hanyga, Multidimensional solutions of time-fractional diffusion-wave equations, Proc. R. Soc. Lond. A 458 (2002) 933-957.

[21] F. Mainardi, G. Pagnini, R. Gorenflo, Mellin transform abd subordination laws in fractional diffusion processes, Fract. Calc. Appl. Anal. 6 (2003) 441-459.

[22] S. J. Osher, J. A. Sethian, Fronts propagating with curvature dependent speed: algorithms based on Hamilton-Jacobi formulations, J. Comput. Phys. 79 (1988) 12-49.

[23] N. Peters, Turbulent Combustion, Cambridge University Press, Cambridge, 490 2004.

[24] V. Mallet, D. E. Keyes, F. E. Fendell, Modeling wildland fire propagation with level set methods, Comput. Math. Appl. 57 (2009) 1089-1101.

[25] E. Jettestuen, J. O. Helland, M. Prodanović, A level set method for simulating capillary-controlled displacements at the pore scale with nonzero contact angles, Water Resour. Res. 49 (2013) 4645-4661.

[26] M. Machacek, G. Danuser, Morphodynamic profiling of protrusion phenotypes, Biophys. J. 90 (2006) 1439-1452.

[27] W. Guo, H. H. Sawin, Review of profile and roughening simulation in microelectronics plasma etching, J. Phys. D: Appl. Phys. 42 (2009) 194014. 
[28] J. J. Monaghan, Smoothed particle hydrodynamics, Rep. Prog. Phys. 68 (2005) 1703-1759.

[29] V. L. Zimont, Gas premixed combustion at high turbulence. turbulent flame closure combustion model, Exp. Therm. Fluid Sci. 21 (2000) 179-186.

[30] V. A. Sabelnikov, A. N. Lipatnikov, Towards an extension of TFC model of premixed turbulent combustion, Flow Turbul. Combust. 90 (2013) 387-400.

[31] T. Ohta, D. Jasnow, K. Kawasaki, Universal scaling in the motion of random interfaces, Phys. Rev. Lett. 49 (1982) 1223-1226.

[32] H. M. Soner, N. Touzi, A stochastic representation for the level set equations, Commun. Part. Diff. Eq. 27 (2002) 2031-2053.

[33] O. Juan, R. Keriven, G. Postelnicu, Stochastic motion and the Level Set Method in Computer Vision: Stochastic active contours, Int. J. Comput. Vision 69 (2006) 7-25.

[34] Y. L. Klimontovich, Nonlinear Brownian motion, Physics-Uspekhi 37 (1994) 737-767.

[35] M. S. Waterman, D. E. Whiteman, Estimation of probability densities by empirical density functions, Int. J. Math. Educ. Sci. Technol. 9 (1978) 127137.

[36] W. R. Schneider, W. Wyss, Fractional diffusion and wave equations, J. Math. Phys. 30 (1) (1989) 134-144.

[37] F. Mainardi, Fractional relaxation-oscillation and fractional diffusion-wave phenomena, Chaos Solitons Fract. 7 (1996) 1461-1477.

[38] F. Mainardi, The fundamental solutions for the fractional diffusion-wave equation, Appl. Math. Lett. 9 (6) (1996) 23-28.

[39] F. Mainardi, Y. Luchko, G. Pagnini, The fundamental solution of the space$153-192$. 
[40] F. Mainardi, G. Pagnini, The Wright functions as solutions of the timefractional diffusion equation, Appl. Math. Comput. 141 (1) (2003) 51-62.

[41] F. Mainardi, G. Pagnini, R. Gorenflo, Some aspects of fractional diffusion equations of single and distributed order, Appl. Math. Comput. 187 (2007) 295-305.

[42] R. Gorenflo, J. Loutchko, Y. Luchko, Computation of the Mittag-Leffler function $E_{\alpha, \beta}(z)$ and its derivative, Fract. Calc. Appl. Anal. 5 (2002) 491518.

[43] E. Jones, T. Oliphant, P. Peterson, et al., SciPy: Open source scientific tools for Python (2001-).

URL http://www.scipy.org/

[44] F. Mainardi, R. Gorenflo, On Mittag-Leffler-type functions in fractional evolution processes, J. Comput. Appl. Math. 118 (2000) 283-299.

[45] J. D. Hunter, Matplotlib: A 2D graphics environment, Comput. Sci. Eng. 9 (3) (2007) 90-95.

[46] F. Pérez, B. E. Granger, IPython: a system for interactive scientific computing, Comput. Sci. Eng. 9 (3) (2007) 21-29. doi:10.1109/MCSE. 2007. 53.

URL http://ipython.org 